\title{
CAPÍTULO 01: CARACTERIZAÇÃO DE CUPCAKES ELABORADOS COM DIFERENTES CONCENTRAÇÕES DE FARINHA DE CASCA DE GUAVIRA
}

\section{CHAPTER 01: CHARACTERIZATION OF CUPCAKES PREPARED WITH GUAVIRA SKIN FLOUR IN DIFFERENT CONCENTRATIONS}

\author{
João Renato de Jesus Junqueira ${ }^{1}$; Mariana Tallita Gomes dos Santos ${ }^{2}$; Danielle Bogo ${ }^{3}$; Ana Cristina
} Araújo Ajalla ${ }^{4}$; Raquel Pires Campos ${ }^{5}$;

\begin{abstract}
Resumo
Cupcakes são produtos de panificação consumidos no Brasil e aceitos por maior parte da população. O objetivo do trabalho foi elaborar a farinha de casca de guavira (FCG), e aplicála na formulação de cupcakes. Na FCG, foram realizadas as análises de umidade $(9,11 \pm 0.81$ $\mathrm{g} / 100 \mathrm{~g})$, cinzas $(2,16 \pm 0.00 \mathrm{~g} / 100 \mathrm{~g})$ e proteínas $(3,54 \pm 0.09 \mathrm{~g} / 100 \mathrm{~g})$. Os cupcakes foram elaborados com diferentes concentrações de FCG (10\% e 15\%) substituindo parcialmente a farinha de trigo. As análises realizadas nos cupcakes foram umidade, cinzas, proteínas, acidez titulável, pH, volume aparente e cor. Os cupcakes com FCG apresentaram maior teor de umidade. Cinzas e proteínas não mostraram diferença significativa $(p<0,05)$ entre as formulações. Acidez titulável apresentou maior teor e $\mathrm{pH}$ menor teor nas formulações com $10 \%$ e $15 \%$ de FCG, quando comparadas com o padrão. O volume aparente diminuiu na presença de FCG e notou-se escurecimento na análise de cor dos cupcakes. De acordo com as análises realizadas, observou-se a viabilidade da substituição parcial de farinha de trigo por FCG na formulação dos cupcakes.
\end{abstract}

Palavras-Chaves: Campomanesia ssp., Cerrado brasileiro, Frutos nativos.

\begin{abstract}
Cupcakes are bakery products consumed in the Brazil and accepted by most of the population. The aim of this work was to obtain guavira skin flour (GSF), and to apply it in the cupcake formulation. For the guavira skin flour, the moisture, ash and protein contents was $9.11 \pm$ $0.81,2.16 \pm 0.00$ and $3.54 \pm 0.09 \mathrm{~g} / 100 \mathrm{~g}$, respectively. The cupcakes were prepared with different concentrations of GSF $(10 \%$ and $15 \%)$ replacing wheat flour. The analyzes performed on the cupcakes were moisture, ash, proteins, titratable acidity, $\mathrm{pH}$, apparent volume and color. Cupcakes prepared with GSF presented higher moisture content. No significant difference $(\mathrm{p}<0.05)$ were observed for the ash and protein contents amid the different formulations. Titratable acidity showed higher content and lower $\mathrm{pH}$ in the formulations with $10 \%$ and $15 \%$ of addition of SGF, in comparison with the standard. The apparent volume decreased in the presence of SGF and color darkening was noted in the cupcakes. According to the performed analyzes, the feasibility of partially replacing wheat flour with SGF in the formulation of cupcakes was observed.
\end{abstract}

Keywords: Campomanesia ssp., Brazilian Savannah, Native fruits.

\footnotetext{
${ }^{1}$ Tecnologia em Alimentos, Universidade Federal de Mato Grosso do Sul, joao.junqueira@ufms.br

2 Tecnologia em Alimentos, Universidade Federal de Mato Grosso do Sul, maritallita95@gmail.com

${ }^{3}$ Tecnologia em Alimentos, Universidade Federal de Mato Grosso do Sul, danielle.bogo@ufms.br

4 Agronomia, Agência de Desenvolvimento Agrário e Extensão Rural de Mato Grosso do Sul, anajallaagraer@gmail.com

${ }^{5}$ Tecnologia em Alimentos, Universidade Federal de Mato Grosso do Sul, raquel.campos@ufms.br
} 


\section{Introdução}

A guavira (Campomanesia ssp.), também conhecida como gabiroba, pertence à família Myrtaceae, sendo um fruto nativo do Cerrado e muito comum no estado de Mato Grosso do Sul. O fruto apresenta reduzida vida útil, com seis dias quando mantido em condições ambiente e sem uso de embalagens adequadas (CAMPOS et al., 2012). A polpa do fruto, de sabor adocicado, pode ser consumida in natura ou em forma de preparações culinárias como geleias e sucos.

Apresenta baixo teor energético, devido à reduzida concentração de macronutrientes, especialmente lipídios $(0,12 \mathrm{~g} / 100 \mathrm{~g})$, e contém bons conteúdos de cálcio, zinco, ferro e fibras (SILVA et al., 2008). Sua polpa e casca são ricas em vitamina C, minerais e compostos bioativos. O bagaço constituído de casca, restos de polpa e sementes da guavira após secagem e pulverização gera a farinha de bagaço de guavira, pode ser utilizado como suplemento alimentar com efeito antitumoral e imunomodulador (ZULIM, 2019).

As farinhas podem ser obtidas de diferentes origens botânicas, embora a farinha de trigo branca seja amplamente utilizada na obtenção de diversos produtos, como bolos, pães e biscoitos. No Brasil, são observados programas de elaboração de alimentos que visam a substituição ou redução da farinha de trigo por outras farinhas obtidas a partir do aproveitamento de partes de plantas e frutos que seriam descartados.

O uso de resíduos de guavira (casca, semente, pedúnculo), provenientes da indústria de polpas congeladas, em adição de até $8 \%$ em barra de cereais foi bem aceito pelas crianças, obtendo-se aceitação sensorial semelhante ao produto padrão e também colabora com a redução de perdas e de efeitos negativos gerados pelo descarte de lixo orgânico no meio ambiente (CAIN et al., 2019).

O desenvolvimento de pesquisas que auxiliem na obtenção de novos produtos com características diferenciadas é uma das necessidades da indústria de alimentos. Os produtos de panificação como bolos e cupcakes são consumidos em todo o mundo, apresentando grande aceitação pela população. De fácil preparo e diversificação de sabores, também possibilitam a substituição total ou parcial de alguns ingredientes, ou adição de componentes que proporcionem melhores características tecnológicas, e forneçam substâncias benéficas ao consumidor (LEBESI; TZIA, 2011).

O melhor aproveitamento dos frutos da guavira associado a criação de novos produtos de panificação pode contribuir com o desenvolvimento da economia do estado de Mato Grosso do Sul e com a sua conservação. Em 2017, este fruto que apresenta importância cultural, foi eleito como símbolo do estado, sendo protagonista de diversos eventos 
gastronômicos, como o "Cata Guavira", em Bonito - MS, que ocorre anualmente e apresenta o apelo para a importância e necessidade do uso sustentável dos frutos nativos, além do Seminário Estadual da Guavira promovido por várias instituições.

Sendo assim, o presente trabalho teve como objetivo avaliar a influência da substituição parcial da farinha de trigo pela farinha de casca de guavira (FCG) em diferentes concentrações (10\% e 15\%) na elaboração de cupcakes, a partir da determinação de propriedades físico-químicas da farinha e dos cupcakes, assim como dos parâmetros de cor e volume aparente dos cupcakes desenvolvidos.

\section{Material e Métodos}

\section{Materiais}

Os frutos de guavira foram coletados no Centro de Pesquisa (Cepaer) da Agência de Desenvolvimento Agrário e Extensão Rural (Agraer), no município de Campo Grande - MS, no mês de novembro de 2019. Na Unidade de Tecnologia em Alimentos (Unital) da Universidade Federal do Mato Grosso do Sul (UFMS), os frutos de guavira foram inicialmente lavados em água corrente. Procedeu-se a separação manual entre a casca e $\mathrm{polpa} / \mathrm{semente}$.

As frações foram congeladas a $-18{ }^{\circ} \mathrm{C}$ para posterior utilização. Para obtenção da farinha da casca da guavira (FCG), as cascas foram descongeladas e em seguida submetidas à secagem em estufa com circulação de ar natural (Lucadema, 82/882, Brasil) a $60{ }^{\circ} \mathrm{C}$, por aproximadamente 48 horas. As cascas desidratadas foram acondicionadas em recipiente de plástico, identificadas e armazenadas, sendo em seguida trituradas em moinho (Tecnal, TE631, Brasil), para obtenção da farinha (Figura 1).

Figura 1. (a) Casca de guavira congelada; (b) Casca de guavira desidratada; (c) Casca de guavira triturada (farinha)

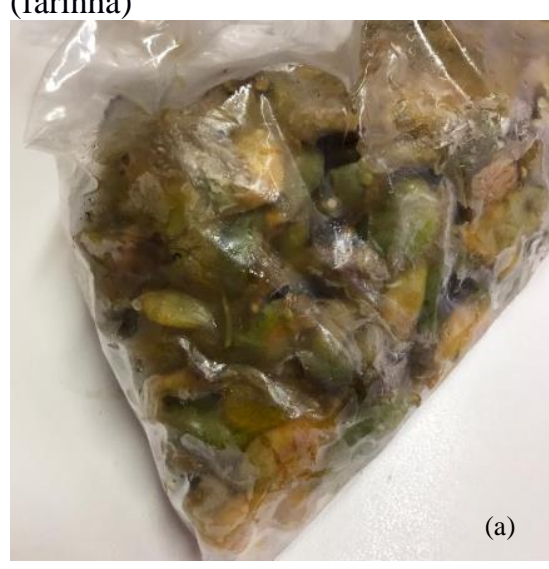

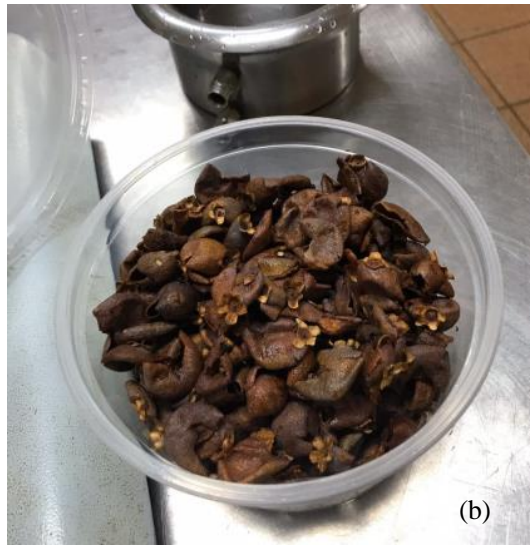

Fonte: Própria (2020).

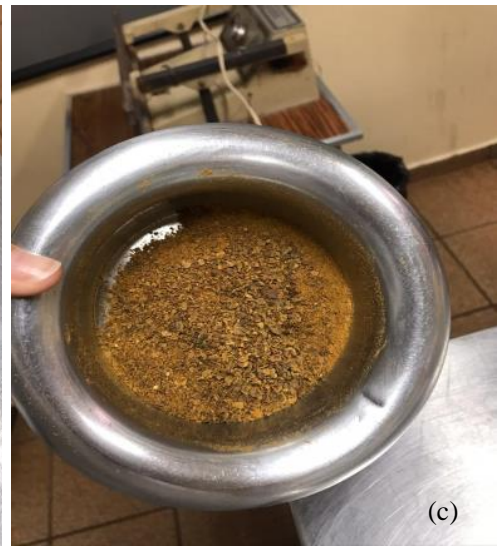


As farinhas de casca de guavira foram identificadas, acondicionadas em recipiente de plástico e armazenadas a temperatura ambiente, por $24 \mathrm{~h}$ até a sua utilização. Os demais ingredientes utilizados na formulação dos cupcakes (farinha de trigo, óleo de soja, açúcar cristal, fermento em pó e ovos) foram adquiridos no comércio local de Campo Grande - MS.

\section{Preparo das formulações}

Foram elaborados três tipos de formulação de cupcakes: Formulação 1 (padrão, sem substituição de farinha de trigo), Formulação 2 (substituição parcial da farinha de trigo pela FCG - 10\%), e Formulação 3 (substituição parcial da farinha de trigo pela FCG - 15\%), conforme apresentado na Tabela 1.

Tabela 1. Formulações dos cupcakes a partir da adição de Farinha de Casca de Guavira (FCG).

\begin{tabular}{cccc}
\hline Ingredientes & Formulação 1 (0\%) & Formulação 2(10\%) & Formulação 3 (15\%) \\
\hline Farinha de trigo & $120 \mathrm{~g}$ & $108 \mathrm{~g}$ & $102 \mathrm{~g}$ \\
Leite & $80 \mathrm{~mL}$ & $80 \mathrm{~mL}$ & $80 \mathrm{~mL}$ \\
Óleo & $40 \mathrm{~mL}$ & $40 \mathrm{~mL}$ & $40 \mathrm{~mL}$ \\
Açúcar & $100 \mathrm{~g}$ & $100 \mathrm{~g}$ & $100 \mathrm{~g}$ \\
Ovos & $44 \mathrm{~g}$ & $44 \mathrm{~g}$ & $44 \mathrm{~g}$ \\
Fermento em pó & $5 \mathrm{~g}$ & $5 \mathrm{~g}$ & $5 \mathrm{~g}$ \\
FCG & - & $12 \mathrm{~g}$ & $18 \mathrm{~g}$ \\
\hline
\end{tabular}

Fonte: Própria (2020).

Na elaboração dos cupcakes, foram adicionados os seguintes ingredientes: farinha de trigo, FCG, óleo de soja, açúcar cristal, leite, fermento em pó e ovos. A preparação da massa dos cupcakes foi realizada manualmente pela mistura contínua das matérias-primas (pesadas em balança analítica), por aproximadamente três minutos, até a obtenção de massa homogênea (Fig. 2). As massas formadas foram dispostas em formas próprias e assadas em forno industrial, na temperatura de $180{ }^{\circ} \mathrm{C}$, de 35 a 40 minutos. Após serem assados, os mesmos foram resfriados, acondicionados em sacos plásticos e armazenados em temperatura ambiente, por $16 \mathrm{~h}$ até a realização das análises.

\section{Caracterização da FCG e dos cupcakes}

A caracterização físico-química da FCG e dos cupcakes foi efetuada no Laboratório de Físico-Química da Unidade de Tecnologia em Alimentos e Saúde Pública (UTASP) da Universidade Federal de Mato Grosso do Sul (UFMS).

A determinação de umidade foi realizada em estufa $\left(105{ }^{\circ} \mathrm{C}\right)$ até peso constante, enquanto a determinação de cinzas foi realizada em mufla $\left(550{ }^{\circ} \mathrm{C}\right)$. Para a análise de proteínas totais, foram avaliados os teores de nitrogênio total, utilizando- se o método 
Kjeldahl empregando como fator de conversão de nitrogênio o valor de 6,25 (IAL, 2008).

Figura 2. Massas dos cupcakes antes do assamento (a) com adição de FCG (b) sem adição de FCG

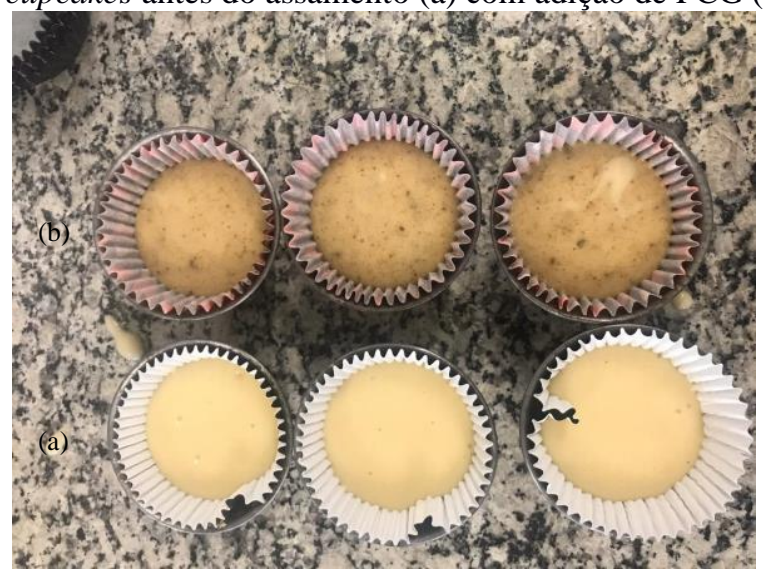

Fonte: Própria (2020).

A composição físico-química das formulações de cupcakes, assim como da FCG foram determinadas segundo metodologia proposta pelo Instituto Adolf Lutz (IAL, 2008), com resultados expressos em g/ $100 \mathrm{~g}$. Todas as análises foram efetuadas em triplicata.

Nas formulações de cupcake, o pH e a acidez titulável, foram avaliados de acordo com as metodologias do Instituto Adolf Lutz (IAL, 2008). O volume aparente dos cupcakes foi determinado pelo deslocamento de sementes de painço, de acordo com método AACC (2000).

A cor da crosta e do miolo dos cupcakes foi determinada empregando colorímetro (Konica Minolta, CM - 2300d, Japão). Os valores de L* (luminosidade, 0 - preto/ 100 branco), $\mathrm{a}^{*}$ (componente vermelho-verde) e $\mathrm{b}^{*}$ (componente amarelo-azul) foram obtidos, sendo a equação 1 utilizada para o cálculo da diferença total de cor $(\Delta \mathrm{E})$.

$$
\Delta E=\sqrt{\left(L^{*}-L_{0}^{*}\right)^{2}+\left(a^{*}-a_{0}^{*}\right)^{2}+\left(b^{*}-b_{0}^{*}\right)^{2}}
$$

O subscrito 0 indica valores obtidos na formulação padrão (sem FCG).

\section{Análises estatísticas}

Os resultados foram analisados utilizando o software Sisvar ${ }^{\circledR}$ (FERREIRA, 2019). Os dados obtidos nas análises físico-químicas e físicas dos cupcakes foram avaliados aplicandose teste de medias (Tukey), com 5\% de nível de significância.

\section{Resultados e Discussão}

\section{Caracterização físico-química da FCG}

As médias e o desvio-padrão dos parâmetros umidade, cinzas e proteínas totais da 
FCG são apresentadas na Tabela 2.

Tabela 2. Composição físico-química da Farinha da casca de Guavira (FCG)

\begin{tabular}{cc}
\hline Componentes & FCG $(\mathrm{g} / 100 \mathrm{~g})$ \\
\hline Umidade & $9,11 \pm 0,81$ \\
Proteínas Totais & $3,54 \pm 0,09$ \\
Cinzas & $2,16 \pm 0,00$ \\
Valores expressos em média \pm desvio padrão. \\
\multicolumn{2}{c}{ Fonte: Própria (2020). }
\end{tabular}

Com base na Tabela 2, observou-se que a FCG obteve teor de umidade igual a 9,11 \pm 0,81 g/ $100 \mathrm{~g}$, abaixo do valor máximo preconizado para farinhas (15 g/ $100 \mathrm{~g}$ ), de acordo com a Resolução RDC n 263 (BRASIL, 2005), o que pode contribuir para manutenção de sua vida útil, bem como retardar o desenvolvimento de microrganismos. Guimarães et al. (2010) obtiveram valor de umidade da farinha da entrecasca da melancia igual a 9,06 g / 100 g, valor próximo ao encontrado em nosso estudo.

O teor de proteínas totais obtido na FCG foi de 3,54 \pm 0,09 g/ $100 \mathrm{~g}$. Cavalcanti et al. (2011) obtiveram o valor de proteínas de 5,57 g/ 100 g para a farinha de juazeiro, valor superior ao encontrado no nosso estudo.

De acordo com a Tabela 2, o teor de cinzas foi igual a 2,16 $\pm 0,00 \mathrm{~g} / 100 \mathrm{~g}$. Campos et al. (2016) encontrou o valor de 2,40 g/ 100 g para cinzas na farinha da casca de pequi, semelhante ao valor reportado para a FCG.

\section{Caracterização físico-química dos cupcakes}

$\mathrm{Na}$ Tabela 3, estão representadas as médias dos componentes físico-químicos analisados das formulações de cupcake.

Tabela 3. Composição físico-química das formulações de cupcakes com diferentes concentrações de Farrinha da Casca de Guavira (FCG) em substituição a farinha de trigo.

\begin{tabular}{cccc}
\hline Componentes & Formulação 1 $(0 \%)$ & Formulação 2 (10\%) & Formulação 3 (15\%) \\
\hline Umidade $(\mathrm{g} / 100 \mathrm{~g})$ & $21,17^{\mathrm{c}} \pm 0,10$ & $22,70^{\mathrm{a}} \pm 0,10$ & $22,00^{\mathrm{b}} \pm 0,01$ \\
Cinzas $(\mathrm{g} / 100 \mathrm{~g})$ & $0,95^{\mathrm{a}} \pm 0,00$ & $0,94^{\mathrm{a}} \pm 0,00$ & $0,99^{\mathrm{a}} \pm 0,10$ \\
Proteínas Totais $(\mathrm{g} / 100 \mathrm{~g})$ & $6,13^{\mathrm{a}} \pm 1,39$ & $6,29^{\mathrm{a}} \pm 0,04$ & $6,20^{\mathrm{a}} \pm 0,39$ \\
Acidez titulável $(\%)$ & $1,13^{\mathrm{b}} \pm 0,05$ & $1,48^{\mathrm{ab}} \pm 0,15$ & $1,82^{\mathrm{a}} \pm 0,27$ \\
pH & $8,80^{\mathrm{a}} \pm 0,05$ & $8,38^{\mathrm{b}} \pm 0,03$ & $8,02^{\mathrm{c}} \pm 0,11$ \\
\hline
\end{tabular}

Letras diferentes na linha indicam diferença significativa pelo teste de Tukey $(\mathrm{p}<0,05)$. Valores expressos em média \pm desvio padrão.

Fonte: Própria (2020).

De acordo com a Tabela 3, observou-se diferença significativa $(\mathrm{p}<0,05)$ nos teores de umidade dos diferentes tratamentos. Maiores teores de umidade foram encontrados nas 
formulações de 10 e $15 \%$ de adição de FCG. A presença de maior teor de fibras na FCG pode ter contribuído para o aumento dos teores de umidade dos cupcakes.

Guimarães et al. (2010) observaram aumento no teor de umidade de bolos adicionados com farinha da entrecasca da melancia. A formulação controle apresentou teor de 25,68 g/ $100 \mathrm{~g}$ e as formulações com adição da farinha apresentaram 30,41 g/ $100 \mathrm{~g}$ e 30,44 g/ $100 \mathrm{~g}$. Estes autores justificaram que a presença de fibras auxilia na retenção de água em sua estrutura durante a etapa de cocção, favorecendo assim um maior teor de umidade final no produto.

Da mesma forma, Andrade et al. (2015), durante a elaboração de bolos com farinha desmucilada de taro, relataram umidade de 23,22 g/ $100 \mathrm{~g}$ a 27,17 g/ $100 \mathrm{~g}$, observando que à medida em que a farinha desmucilada de taro era adicionada, o teor de umidade era aumentado, pois as fibras apresentam propriedade de retenção de água.

Pereira et al. (2010 a) obtiveram teores de umidade de bolos adicionados com flocos de amaranto variando entre 23,9 g/ $100 \mathrm{~g}$ a 28,3 g/ $100 \mathrm{~g}$. Estes autores observaram diferenças significativas para este parâmetro nos diferentes tratamentos (0, 10, 20 e 30\%).

A Agência Nacional de Vigilância Sanitária (Anvisa) recomenda valores máximos de $38 \mathrm{~g} / 100 \mathrm{~g}$ de umidade para produtos de panificação, estando o bolo desenvolvido no presente estudo dentro do recomendado (BRASIL, 2000).

O teor de cinzas representa a quantidade de minerais que contém no produto avaliado. Observando a Tabela 3 , percebe-se que não houve diferença significativa $(p>0,05)$ entre as formulações de cupcakes avaliadas. Moscatto et al. (2004) encontraram valores de cinzas variando entre 1,42 a 1,93 g/ $100 \mathrm{~g}$ em formulações de bolos de chocolate com substituição parcial de farinha de trigo por farinha de yacon e inulina em diferentes concentrações. Pereira et al. (2010a) encontraram valor médio de cinzas igual a 1,99 g/ $100 \mathrm{~g}$ em formulações de bolos de com adição de flocos de amaranto em diferentes concentrações.

Não houve diferença significativa $(p>0,05)$ no teor de proteínas entre as formulações de cupcakes, variando de 6,13 a 6,29 g/ 100 g (Tabela 3). Ou seja, a substituição parcial de farinha de trigo pela FCG não altera o valor protéico nos cupcakes. Marcelino et al. (2018) obtiveram valores 7,8 e 8,52 g/ 100 g quando elaboraram cupcakes com diferentes concentrações de farinha de casca e polpa de baru. Durante a elaboração de bolos com adição de flocos de amaranto em diferentes concentrações, Pereira et al. (2010a) obtiveram valores de proteínas variando entre 5,60 a 6,05 g/ 100 g.

Observando o parâmetro acidez titulável, nota-se que houve diferença significativa $(p<0,05)$ entre as formulações padrão e $15 \%$, entretanto, não houve diferença significativa 
( $p>0,05)$ entre a formulação padrão e $10 \%$, e entre $10 \%$ e $15 \%$. Conclui-se que a maior substituição de FCG levou a um aumento na acidez dos cupcakes.

Moura et al. (2014) obtiveram valor próximo de acidez titulável ao analisarem bolo com farinha de pupunha, 2,47\%, e valores superiores em bolos com adição de farinha de açaí $(4,13 \%)$ e farinha de tucumã $(4,13 \%)$. Silva (2019) obteve valores menores, que ficaram entre $0,05 \%$, e $0,24 \%$ em formulações de bolo com substituição parcial da farinha de trigo pela farinha da massa do xiquexique.

Analisando a Tabela 3, houve diferença significativa $(\mathrm{p}<0,05)$ no $\mathrm{pH}$ entre a formulação padrão e as formulações com substituição por FCG. Observou-se que, ao adicionar a FCG, diminui-se o pH dos cupcakes, o que vem ao encontro do valor aumentado de acidez titulável encontrado nestas formulações. Poletto et al. (2015) obtiveram valor menor, 7,05, ao analisarem bolo de chocolate modificado. Maia et al. (2018) relataram pH 6,65 , ao elaborarem bolo de milho com adição de $5 \%$ de farinha de maracujá.

\section{Análises físicas dos cupcakes}

Os parâmetros de cor para crosta e miolo dos cupcakes estão apresentados na Tabela 4.

De acordo com a Tabela 4, foram observadas diferenças significativas entre os diferentes tratamentos $(\mathrm{p}<0,05)$ entre si para os parâmetros de cor. Conforme maior adição da farinha de casca da guavira, as formulações de cupcake apresentavam redução no parâmetro de luminosidade $\left(\mathrm{L}^{*}\right)$, indicando possível escurecimento tanto da crosta quanto do miolo.

Tabela 4. Composição físico-química das formulações de cupcakes, com diferentes concentrações de Farinha da Casca de Guavira (FCG) em substituição a farinha de trigo.

\begin{tabular}{ccccc}
\hline Crosta & \multicolumn{1}{c}{ Formulação 1 $(0 \%)$} & Formulação 2 (10\%) & Formulação 3 $(15 \%)$ \\
\hline & $\mathrm{L}^{*}$ & $7,05^{\mathrm{a}} \pm 0,15$ & $2,47^{\mathrm{b}} \pm 1,08$ & $1,36^{\mathrm{b}} \pm 0,53$ \\
& $\mathrm{a}^{*}$ & $3,68^{\mathrm{a}} \pm 1,82$ & $1,24^{\mathrm{ab}} \pm 0,38$ & $0,88^{\mathrm{b}} \pm 0,47$ \\
& $\mathrm{~b}^{*}$ & $7,62^{\mathrm{a}} \pm 0,67$ & $2,17^{\mathrm{b}} \pm 0,96$ & $1,14^{\mathrm{b}} \pm 0,64$ \\
\hline Miolo & & & \\
\hline & $\mathrm{L}^{*}$ & $13,23^{\mathrm{a}} \pm 6,38$ & $2,32^{\mathrm{b}} \pm 0,51$ & $3,55^{\mathrm{b}} \pm 0,94$ \\
& $\mathrm{a}^{*}$ & $1,06^{\mathrm{a}} \pm 0,27$ & $0,71^{\mathrm{a}} \pm 0,08$ & $1,19^{\mathrm{a}} \pm 0,34$ \\
& $\mathrm{~b}^{*}$ & $8,81^{\mathrm{b}} \pm 3,13$ & $1,67^{\mathrm{a}} \pm 0,36$ & $2,74^{\mathrm{a}} \pm 0,81$ \\
\hline
\end{tabular}

Letras diferentes na linha indicam diferença significativa pelo teste de Tukey $(\mathrm{p}<0,05)$. Valores expressos em média \pm desvio padrão.

Fonte: Própria (2020).

Padilha et al. (2010) ao obterem valores de 20,91 e 19,79 no parâmetro luminosidade $\left(\mathrm{L}^{*}\right)$, observaram que a adição de farinha de yacon em formulações de bolos de chocolate influenciou em sua caracterização cromática, pois deixou-os mais escuros.

De acordo com Pereira et al. (2010b), o parâmetro luminosidade ( $\left.\mathrm{L}^{*}\right)$ variou de 52,2 a 
56,7 para cor da crosta e de 70,9 a 74,2 para cor do miolo em bolos elaborados com diferentes concentrações de flocos de amaranto.

$\mathrm{Na}$ Tabela 4, observando o parâmetro a* da crosta, percebe-se que houve diferença significativa $(\mathrm{p}<0,05)$ entre as formulações. O padrão se mostrou diferente da formulação com $15 \%$ de FCG, porém, igual à formulação com $10 \%$. O parâmetro a ${ }^{*}$ do miolo, não apresentou diferença significativa ( $>$ >0,05) entre as formulações com adição de FCG e o padrão.

Ao observar o parâmetro $b^{*}$ observou-se que as formulações com substituição parcial da farinha de trigo por FCG, apresentaram diferença significativa (p>0,05) quando comparadas ao padrão. Ao aumentar a concentração de FCG nas formulações, o valor de b* foi reduzido, demonstrando que o bolo obteve menos tons de amarelo. As formulações com substituição de 10 e $15 \%$ de FCG não diferiram entre si. No caso de bolos elaborados com farinha de semente de abóbora, Bitencourt et al. (2014) relataram que apresentaram valores de $b^{*}$ maiores, com 27,83 a 31,18, que aqueles com apenas farinha de trigo, 21,82.

A diferença total de cor $(\Delta \mathrm{E})$ da crosta para a formulação $10 \%$ foi de 7,51 , e para a formulação de $15 \%$ foi de 9,06 . Isso indica que maior substituição de farinha de trigo por FCG levou a um aumento na diferença de cor da crosta com relação à formulação padrão.

De forma semelhante Borges et al. (2013) observaram que ao aumentar a concentração de quinoa na formulação de bolo, houve aumento de $\Delta \mathrm{E}$, indicando escurecimento do mesmo. Andrade et al. (2015) também relataram que ao aumentar a concentração de farinha de taro em bolos, os valores de $\Delta \mathrm{E}$ apresentaram aumento quando comparados ao padrão, mostrando diferença na cor entre as formulações.

A Figura 3 apresenta as diferentes formulações de cupcakes. Visualmente, percebe-se diferenças de coloração entre as formulações após o assamento, o que pode ser comprovado através da Tabela 4.

Na Figura 4 são apresentados os valores de volume aparente obtidos das formulações de cupcakes. Observa-se diferença significativa $(\mathrm{p}<0,05)$ entre os cupcakes com substituição parcial de FCG e o com formulação padrão. Os cupcakes com substituição apresentaram menor volume aparente após cocção e não mostraram diferença significativa $(p>0,05)$ entre si. Ainda assim, as formulações com substituição parcial de farinha de casca de guavira, tiveram um bom desempenho de crescimento.

Ozores et al. (2015) observaram que bolos com adição de 5\% e 10\% de farinha de maracujá obtiveram um aumento significativo no volume específico em relação ao padrão, o que não foi observado com a adição da farinha de casca de guavira. Guimarães et al. (2010) ao estudarem a influência da adição de farinha de entrecasca de melancia na elaboração de 
bolos, observaram que ao adicionar $7 \%$ houve aumento no volume, mas ao adicionar 30\%, houve diminuição. Novos estudos podem ser realizados para verificar a redução do volume aparente nos cupcakes com substituição parcial de FCG.

Figura 3. Cupcakes após o assamento (a) Formulação 1 (0\%), (b) Formulação 2 (10\%), (c) Formulação 3 (15\%)

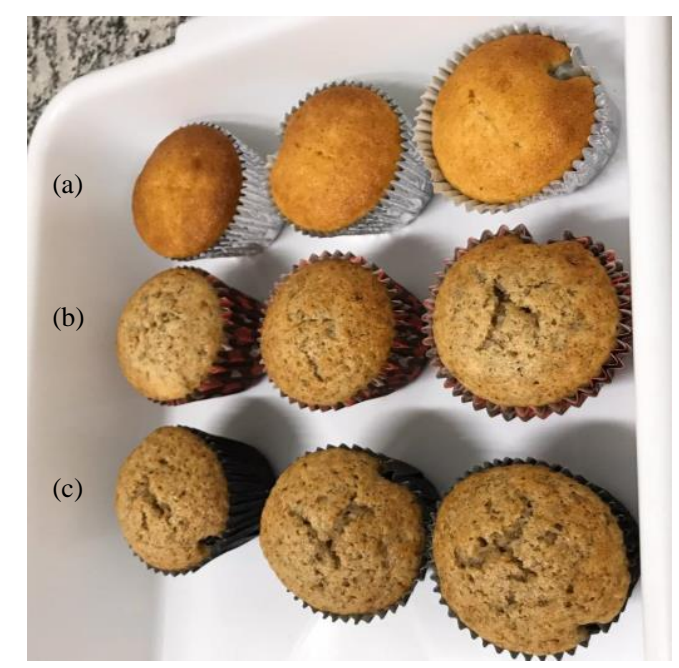

Fonte: Própria (2020).

Figura 4. Valores de volume aparente obtidos das formulações de cupcakes (Médias \pm DP), com diferentes concentrações de Farrinha da Casca de Guavira (FCG) em substituição a farinha de trigo.

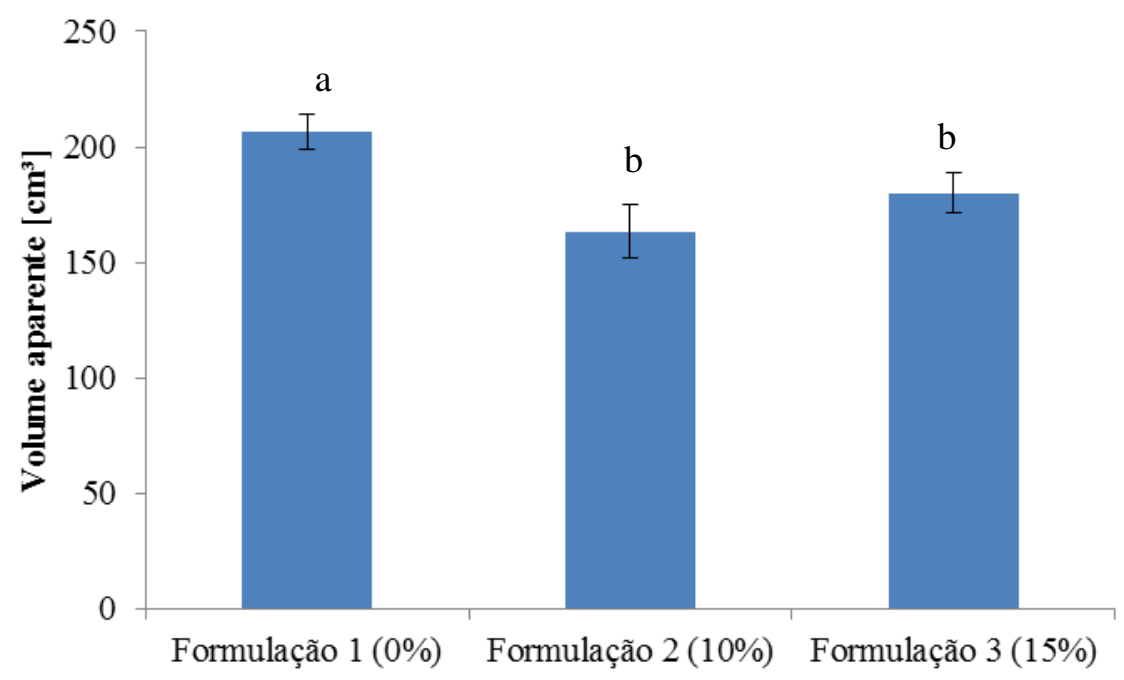

Letras diferentes indicam diferença significativa pelo teste de Tukey $(\mathrm{p}<0,05)$. Valores expressos em média \pm desvio padrão.

Fonte: Própria (2020).

Embora os resultados das análises físicas sejam diferentes do padrão sem adição de FCG, a substituição de farinha de trigo por farinha de casca de guavira apresentou resultados gerais positivos demonstrando a viabilidade do seu uso na elaboração de cupcakes com estes valores de substituição da farinha de trigo. 


\section{Conclusões}

A farinha da casca de guavira (FCG) apresentou baixo teor de umidade, dentro do preconizado para farinhas, com valores de proteínas 3,54 e cinzas de 2,16.

Os cupcakes com substituição de 10 e $15 \%$ de farinha de trigo por FCG apresentaram maior umidade do que a formulação padrão, sem adição de FCG. Os conteúdos de cinzas e proteínas não apresentaram diferença significativa $(\mathrm{p}>0,05)$ entre os cupcakes.

A substituição parcial de farinha de trigo por FCG resultou em maior acidez titulável diminuição do pH em relação ao cupcake padrão.

Conforme o aumento na concentração de FCG maior o escurecimento e menor tonalidade amarelada dos cupcakes, além da diminuição do volume aparente. Conclui-se, pelos resultados obtidos que é viável a substituição de farinha de trigo por farinha de casca de guavira.

\section{Referências}

ANDRADE, L. A.; NAGATA, C. L. P.; ASSUMPÇÃO, G. M. P.; GONÇALVES, G. A. S.; PEREIRA, J. Farinha desmucilada de taro utilizada na elaboração de bolos. Científica, v 43, p. 203-214, 2015

AMERICAN ASSOCIATION OF CEREAL CHEMISTS (AACC). Approved Methods of the AACC. 10. ed. Sant Paul: American Association of Cereal Chemists, 2000.

ASSOCIATION OF OFFICIAL ANALYTICAL CHEMISTS (AOAC). Official Methods of analysis of AOAC International. 19 ed. Washington: AOAC International, 2012.

BITENCOURT, C.; DUTRA, F. L. G.; PINTO, V. Z.; HELBIG, E.; BORGES, L. R. Elaboração de bolos enriquecidos com semente de abóbora: avaliação química, física e sensorial. Boletim Ceppa, v. 32, n. 1, p. 19-32, 2014.

BORGES, J. T. S.; PIROZI, M. R.; VIDIGAL, J. G.; PAULA, C. D.; SILVA, N. A. S. Utilização de farinha mista de trigo e quinoa na elaboração de bolos. Revista Brasileira de Tecnologia Agroindustrial, v. 07, n. 2, p. 1034-1048, 2013.

BRASIL. Ministério de Agricultura, Pecuária e Abastecimento. Instrução normativa $n^{\circ}$ 8, 03 jun. 2005. Regulamento Técnico de Identidade e Qualidade da Farinha de Trigo. Diário Oficial da República Federativa do Brasil, Brasília, DF, Seção 1, n. 105, p. 91.03 junho, 2005 .

CAIN, J. P.; SILVA, A. C. G. da; SOARES, J. M.; SANTOS, M. M. R.; AMARAL, L. A. do; SANTOS, E. F dos; NOVELLO, D. Adição de farinha de resíduos de guavira em barra de cereais: aceitabilidade sensorial e caracterização físico-química. Conexão Ci, Formiga, MG, v. 14 , n. 1, p.18-26. 2019.

CAMPOS, R. P.; HIANE, P. A.; RAMOS, M. I. L.; RAMOS FILHO, M. M.; Macedo, M. L. R. Conservação pós-colheita de guavira (Campomanesia sp.). Revista Brasileira de Fruticultura, Jaboticabal , v. 34, n. 1, p. 41-49, Mar. 2012. 
CAMPOS, R. P. et al. Elaboração e caracterização de farinha da casca de pequi. Agroecologia, v. 11, n. 2, p. 1-12, 2016.

CAVAlCANTI, M. T.; SILVEIRA, D. C.; FLORÊNCIO, I. M.; FEITOSA, V. A.; ELLER, S. C. W. S. Obtenção da farinha do fruto do juazeiro (Ziziphus joazeiro mart.) e caracterização físico-química. Revista Verde de Agroecologia e Desenvolvimento Sustentável, v. 6, p. 220-224, 2011.

FERREIRA, D. F. SISVAR: A Computer Analysis System To Fixed Effects Split Plot Type Designs. Revista Brasileira De Biometria, [S.1.], v. 37, n. 4, p. 529-535, 2019.

GUIMARÃES, R. R.; FREITAS, M. C. J.; SILVA, V. L. M. Bolos simples elaborados com farinha da entrecasca de melancia (Citrullus vulgaris, sobral): avaliação química, física e sensorial. Ciência e Tecnologia de Alimentos, v. 30, n. 2, p. 354-363, 2010.

INSTITUTO ADOLFO LUTZ. Métodos físico-químicos para análise de alimentos. São Paulo: Instituto Adolfo Lutz, 2008. 1020 p.

LEBESI, D. M.; TZIA, C. Effect of the Addition of Different Dietary Fiber and Edible Cereal Bran Sources on the Baking and Sensory Characteristics of Cupcakes. Food and Bioprocess Technology, v. 4, p. 710-722, 2011.

MAIA, S. et al. Farinha de maracujá na elaboração de bolo de milho. Revista Verde de Agroecologia e Desenvolvimento Sustentável, v. 13, n. 3, p. 328-336, 2018.

MARCELINO, G. et al. Caracterização e análise sensorial de cupcakes elaborados com diferentes concentrações de farinha de casca e polpa de baru (Dipteryx alata Vog.). Multitemas, p. 265-281, 2018.

MIRANDA, A. A.; CAIXETA, A. C. A.; FLÁVIO, E. F.; PINHO, L. Desenvolvimento e análise de bolos enriquecidos com farinha da casca do maracujá (Passiflora edulis) como fonte de fibras. Alimentos e Nutrição, v. 24, n. 2, p. 225-232, 2013.

MOSCATTO, J. A.; PRUDÊNCIO, S. H.; HAULY, M. C de O. Farinha de yacon e inulina como ingredientes na formulação de bolo de chocolate. Ciência e Tecnologia de Alimentos, v. 24, n. 4, p. 634-640, 2004.

MOURA, K.; MOURA, S. Desenvolvimento e avaliação das características nutricionais, físico-químicas e sensoriais de bolo com diferentes tipos de farinhas e castanha-dobrasil. Monografia (Bacharelado em Engenharia de Alimentos) - Universidade Federal de Rondônia, Ariquemes, 2014.

OZORES B., et al. Aceitabilidade e características tecnológicas de bolo enriquecido com farinha de maracujá. Revista Ciências da Saúde, v. 16, n. 1, p. 61-69, 2015.

PADILHA, V. M.; ROLIM, P. M.; SALGADO, S. M.; LIVEIRA, A. S.; ANDRADE, S. A. C.; GUERRA, N. B. Perfil sensorial de bolos de chocolate formulados com farinha de yacon (Smallanthus sonchifolius). Ciência e Tecnologia de Alimentos, v. 30, n. 3, p. 735-740, 2010.

PEREIRA, L.; FERNANDES, G. S.; JESUS, J. R.; ALVES, J. S.; PEREIRA, J. Composição 
físico-química de bolos elaborados com amaranto em flocos. XXIV Congresso de Iniciação Científica da Universidade Federal de Lavras, Lavras, 2010a.

PEREIRA, L.; FERNANDES, G. S.; JESUS, J. R.; ALVES, J. S.; PEREIRA, J. Características físicas de bolos elaborados com amaranto em flocos. XXIV Congresso de Iniciação Científica da Universidade Federal de Lavras, Lavras, 2010 b.

POLETTO, B. O.; SANTOS, R. D.; RIBEIRO, E. T. Avaliação físico-química de bolo de chocolate modificado. Revista Científica da Faculdade de Educação e Meio Ambiente, v. 6, n. 2, p. 77-91, 2015.

SILVA, M. R.; LACERDA, D. B. C. L.; SANTOS, G. G.; MARTINS, D. M. DE O. Caracterização química de frutos nativos do Cerrado. Ciência Rural, Santa Maria, v. 38, n. 6, p. 1790-1793, 2008.

SILVA, C. Desenvolvimento, caracterização e análise sensorial de bolo a partir da farinha de xiquexique (Pilosocereus gounellei). XIV Jornada de Iniciação Científica e Inovação Tecnológica, VIII Jornada de Trabalhos de Extensão e VII Jornada de Iniciação a Docência do IF sertão PE. Campus Floresta, Pernambuco, 2019.

ZULIN, N. E. A suplementação com farinha do bagaço do fruto de Campomanesia adamantium promove imunomodulação e redução do crescimento tumoral em ratos Wistar portadores de tumor de Walker 256. Dissertação (mestrado) - Universidade Federal do Paraná, Setor de Ciências Biológicas, Programa de Pós-Graduação em Ciências Fisiológicas. Curitiba, 2019. 